\title{
APLICAÇÃO DO PROCESSO DE PRODUÇÃO ENXUTA NA OPERAÇÃO UNITÁRIA DE FILTRAGEM NO PROCESSO DE PELOTIZAÇÃO DAS USINAS ITABRASCO E HISPANOBRÁS*
}

Ângelo José de Santana Tavares Cavalcanti ${ }^{1}$ Everson Godoy ${ }^{2}$ Henrique Resende ${ }^{3}$ Josiane Lira Batista 4

Resumo Richardson Barcellos ${ }^{6}$

Este trabalho apresenta o desempenho após a implantação do processo de produção enxuta na operação unitária de filtragem do processo de pelotização das usinas Itabasco e Hispanobrás, com o propósito de aumentar a produtividade, reduzir custos, reduzir paradas de usina e perdas de produção, otimizando as rotinas operacionais e a utilização da mão de obra, bem como a melhoria do ambiente de trabalho. Esse processo é pioneiro em regime de produção contínua.

Palavras-chave: Produção enxuta; Filtragem; Processo de pelotização.

\section{APPLICATION OF LEAN MANUFACTURING PROCESS IN OPERATION UNIT FILTER IN CASE OF PELLET OF PLANTS ITABRASCO AND HISPANOBRAS}

\begin{abstract}
This paper show the performance after the implementation of lean manufacturing process in filtration unit operation of the process of pelletizing Itabasco Hispanobrás and plants, in order to increase productivity, reduce costs, reduce plant stoppages and production losses, optimizing operational routines and the use of labor as well as improving the working environment. This process is a pioneer in continuous production system.
\end{abstract}

Keywords: Lean production; Filtering; Pelletizing process.

\footnotetext{
Engenheiro de Materiais, Engenheiro, Engenheiro de Processo, DIPE, VALE, Vitoria, ES, Brasil. Engenheiro, Analista Sênior de Processos, DIPE, VALE, Vitoria, ES, Brasil.

Engenheiro, Analista Sênior de Processos, DIPE, VALE, Vitoria, ES, Brasil.

4 Engenheiro de Produção, Engenheira, Supervisora de Rotina Operacional, DIPE, VALE, Vitoria, ES, Brasil.

5 Técnico, Técnico Especialista, DIPE, VALE, Vitoria, ES, Brasil.
}

\footnotetext{
*Contribuição técnica ao 44 Seminário de Redução de Minério de Ferro e Matérias-primas, $15^{\circ}$ Simpósio Brasileiro de Minério de Ferro e 2ํㅗ Simpósio Brasileiro de Aglomeração de Minério de Ferro, 15 a 18 de setembro de 2014, Belo Horizonte, MG, Brasil.
} 


\section{INTRODUÇÃO}

A pelotização é um processo de aglomeração que, através de um tratamento térmico converte a fração ultrafina em esferas de tamanho da faixa de 8 a $18 \mathrm{~mm}$, possuindo características apropriadas para a alimentação nas unidades de redução [1].

As etapas envolvidas no processo de pelotização podem, de forma genérica, ser agrupadas em três estágios:

- preparação das matérias-primas;

- formação das pelotas cruas;

- processamento térmico.

A preparação das matérias primas tem por objetivo adequar as características do minério de ferro (pellet feed) às exigidas para a produção de pelotas cruas. Neste estágio é preparada a mistura a pelotizar, que pode comportar diferentes tipos de minérios e aditivos, estes utilizados para modificar a composição química e as propriedades metalúrgicas das pelotas. Em geral, incluem-se neste estágio as seguintes etapas: concentração / separação, homogeneização das matérias primas, moagem, classificação, espessamento, homogeneização da polpa e filtragem.

A formação de pelotas cruas, também conhecida por pelotamento, tem por objetivo produzir pelotas numa faixa de tamanhos apropriada e com resistência mecânica suficiente para suportar as etapas de transferência e transporte entre os equipamentos de pelotamento e o de tratamento térmico.

A fim de conferir às pelotas alta resistência mecânica e características metalúrgicas apropriadas, as mesmas são submetidas a um processamento térmico, que envolve etapas de secagem, pré-queima, queima, pós-queima e resfriamento. O tempo de duração de cada etapa e a temperatura a que são submetidas as pelotas têm forte influência sobre a qualidade do produto final.

Um fluxograma típico do processo de pelotização das usinas da CVRD é apresentado na figura [1-2].

\section{FLUXX DO PROCESSO}

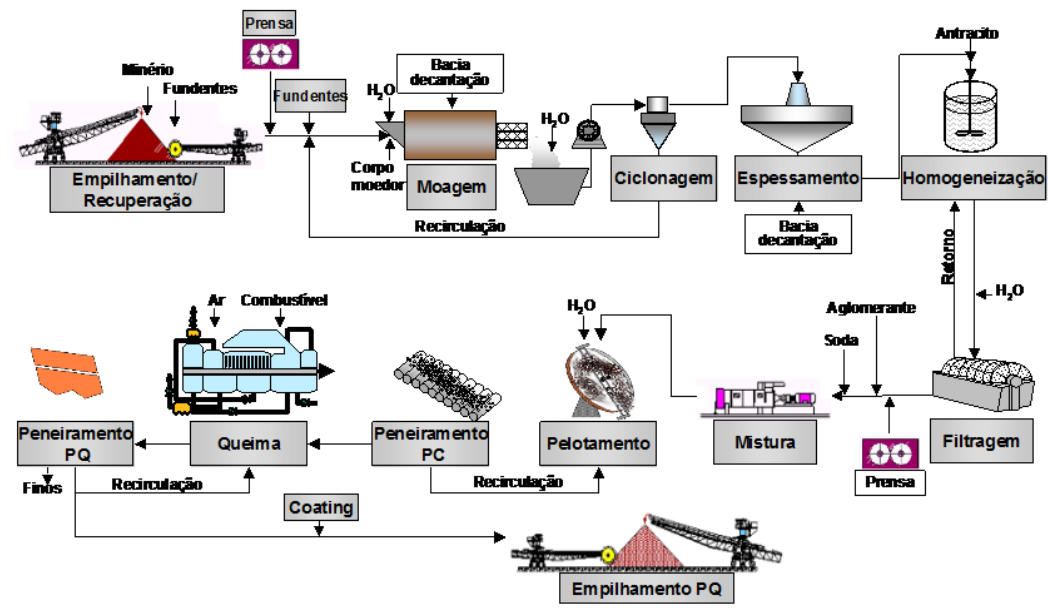

Figura 1. Fluxograma do processo de pelotização de minério de Ferro.

O atual nível competitivo mundial tem feito empresas dos mais variados segmentos buscarem aumentar sua competitividade utilizando estratégias que valorizem atributos como custos e qualidade [3]. Isso faz com que as empresas industriais que utilizam a abordagem de produção enxuta gozem, em geral, de uma vantagem competitiva sobre as que utilizam abordagens tradicionais, conforme tem sido percebido pela

\footnotetext{
*Contribuição técnica ao 44 Seminário de Redução de Minério de Ferro e Matérias-primas, $\quad$ 15은 Simpósio Brasileiro de Minério de Ferro e 2ํㅗ Simpósio Brasileiro de Aglomeração de Minério de Ferro, 15 a 18 de setembro de 2014, Belo Horizonte, MG, Brasil.
} 
indústria e também pela academia, não só nos países desenvolvidos, como também nos emergentes [4].

O just-in-time (JIT) é baseado na lógica de que nada deve ser produzido até que seja necessário [5]. A abordagem JIT foi inicialmente desenvolvida na Toyota Motor Company of Japan pelos engenheiros Eiigi Toyoda e Taiichi Ohno.

O sistema desenvolvido tinha o objetivo de produzir vários modelos de automóvel, em pequenas quantidades, porém de maneira eficiente e lucrativa. O JIT envolveria, então, o fluxo contínuo e em lotes pequenos de peças para atender o cliente na hora exata, na quantidade e local corretos, exigindo perfeita conexão e sincronia entre todos os processos, fornecedores e distribuidores.

De acordo com Womack, Jones e Roos [6], o termo lean production foi utilizado pela primeira vez por Krafcik, referindo-se aos processos JIT. O termo world class manufacturing, também muito utilizado para referir-se a sistemas abrangentes de produção enxuta, foi utilizado pela primeira vez por Hayes e Wheelwright em 1984 [7]. A produção enxuta é uma filosofia produtiva essencialmente utilizada com o objetivo de eliminar ou minimizar atividades não agregadoras de valor ao produto final, a Produção Enxuta também é conhecida como TPS (Toyota Production System ou em português - Sistema Toyota de Produção), Lean Manufacturing, Produção Lean ou ainda Lean Thinking.

Atualmente, essa filosofia encontra-se difundida por todas as partes e está se transformando em um elemento essencial na manutenção da competitividade das empresas que, independente do porte, veem nesta prática um caminho assertivo na busca da maior qualidade e do menor custo.

Após identificar as oportunidades de melhoria com a implantação deste programa, decidiu-se inovar e implantá-lo num processo de produção contínua, visto que preferencialmente é aplicado em cadeia automotiva. Este estudo apresenta o desempenho após a implantação do processo de produção enxuta na operação unitária de filtragem cujo objetivo foi aumentar a produtividade, reduzir custos, reduzir paradas de usina e perdas de produção, otimizando as rotinas operacionais e a utilização da mão de obra.

\section{MATERIAIS E MÉTODOS}

Como mencionado anteriormente, o objetivo neste programa foi aumentar a produtividade, padronização das atividades, melhorias de limpeza e organização da área gerando a satisfação das pessoas que atuam neste local.

Após a escolha da etapa de operação unitária do processo de pelotização para implantação do projeto produção enxuta foi definido um macro cronograma com levantamento das variáveis criticas:

1. Planejamento da implantação do Projeto

2. Levantamento de Variáveis Criticas e Restrições do projeto

3. Levantamento e definição de indicadores de controle e registros

4. Planejamento do Work Shop Kaizen por atividade

5. Treinamento inicial com toda equipe da Filtragem (Supervisores, Engenheiros, Técnicos (operação e manutenção), Operadores, Mecânicos e Inspetores.

6. Acompanhamento das atividades com o objetivo de identificar desperdícios e despadronização de atividades

7. Padronização / Análise do processo

8. Fechamento

*Contribuição técnica ao 44 Seminário de Redução de Minério de Ferro e Matérias-primas, $15^{\circ}$ Simpósio Brasileiro de Minério de Ferro e 2ํㅗ Simpósio Brasileiro de Aglomeração de Minério de Ferro, 15 a 18 de setembro de 2014, Belo Horizonte, MG, Brasil. 
Para direcionamento dos passos 5 e 6 foi realizado levantamento das causas da baixa produtividade da filtragem utilizando o método do Ishikawa.

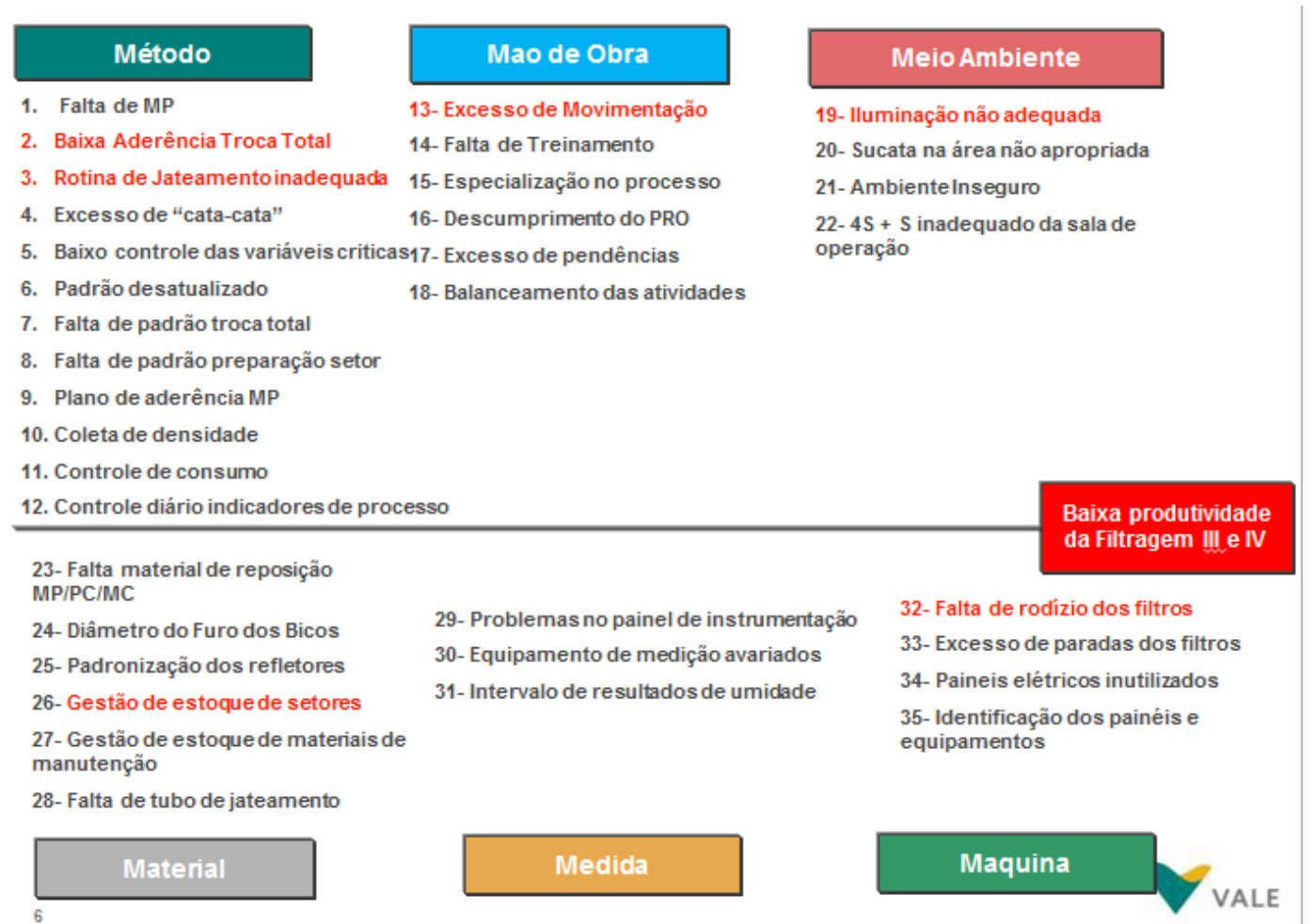

Figura 2. Causas levantadas para baixa produtividade da filtragem.

Inicialmente foram levantadas 35 causas e priorizadas 6 . Como resultados dessas atividades foram propostas 58 Kaizens para as causas levantadas.

\section{RESULTADOS E DISCUSSÃO}

Os dados abaixo demonstram a redução nos resultados de umidade da polpa retida, principal indicador da filtragem, para as usinas Itabrasco e Hispanobrás.

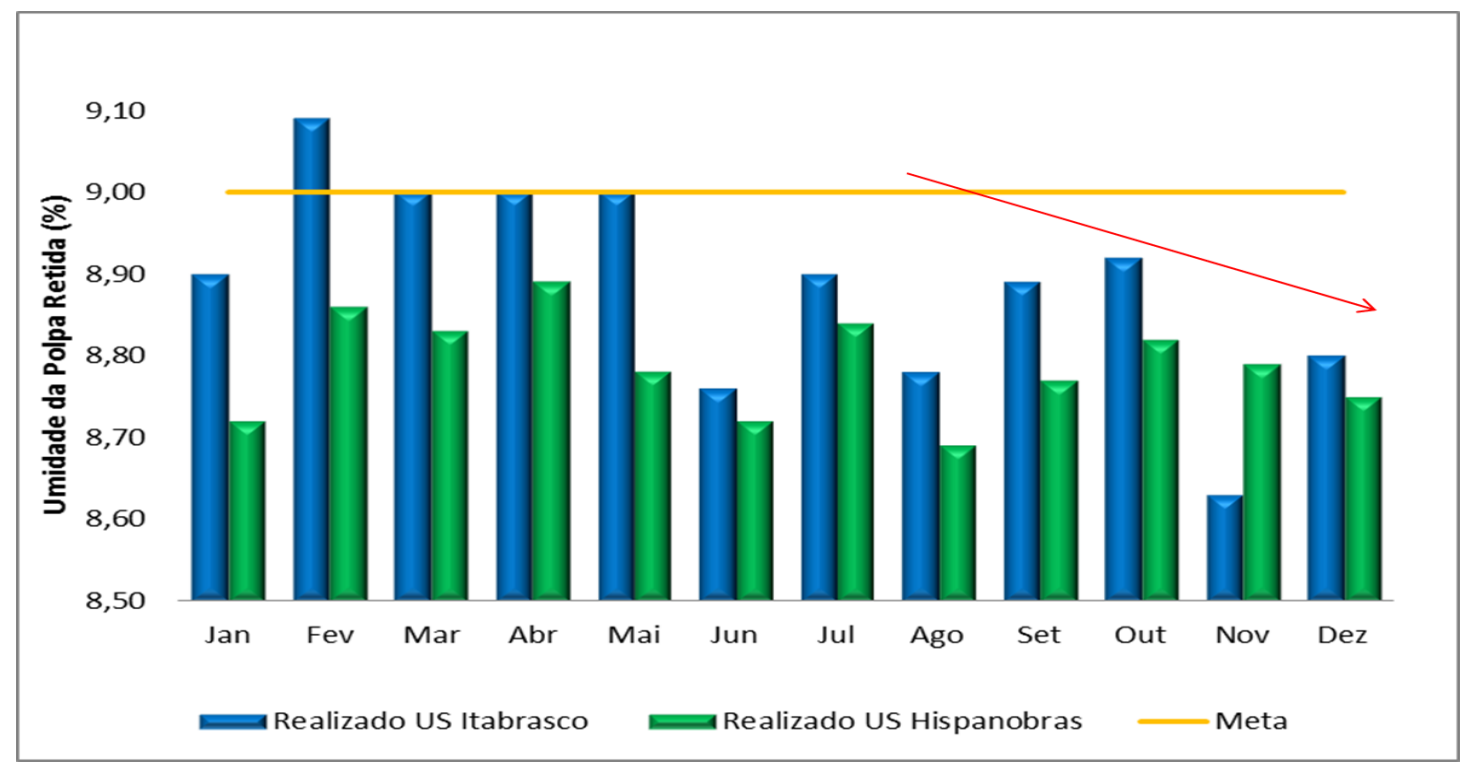

Figura 3. Gráfico evolução da umidade da polpa retida ao longo de 2013.

* Contribuição técnica ao 44ํ Seminário de Redução de Minério de Ferro e Matérias-primas, Simpósio Brasileiro de Minério de Ferro e 2º Simpósio Brasileiro de Aglomeração de Minério de Ferro, 15 a 18 de setembro de 2014, Belo Horizonte, MG, Brasil. 


\subsection{Padronização}

Situação antes: Painel de identificação dos filtros sem preenchimento.

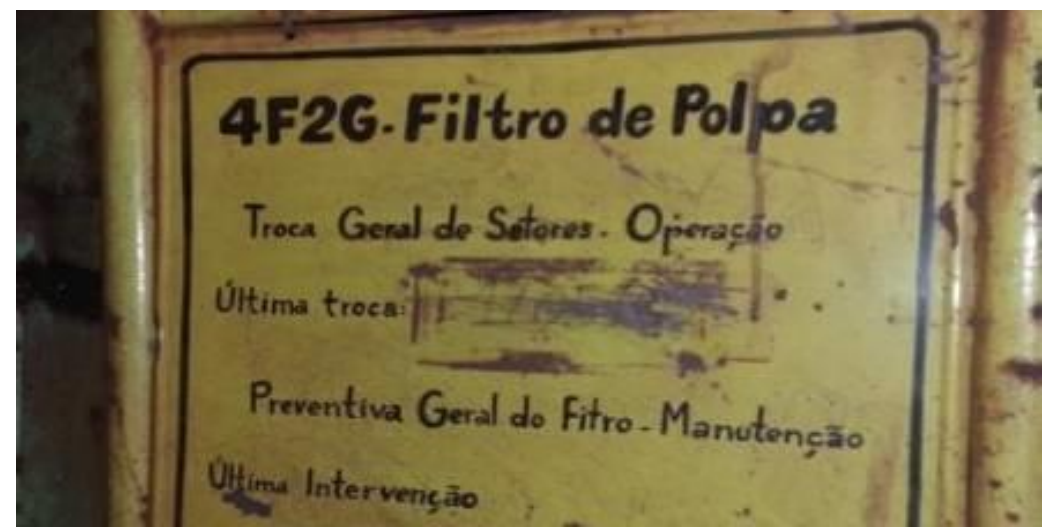

Figura 4. Painel de identificação dos filtros.

Situação Proposta: Utilizar de forma visual somente informações relevantes ao operador.

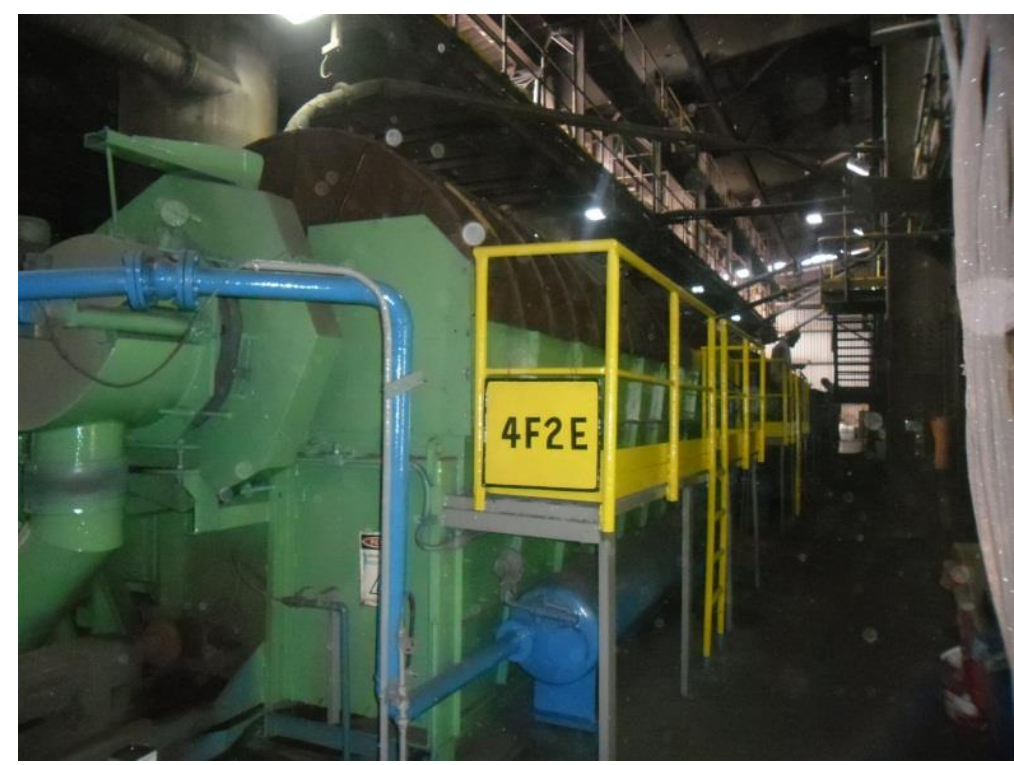

Figura 5. Painel de identificação dos filtros.

Situação antes: Excesso de movimentação do operador para realizar troca de setores. Setores alocados no final da usina longe de algumas linhas.

Algumas vezes, os setores usados deixados escorados próximos aos filtros. 

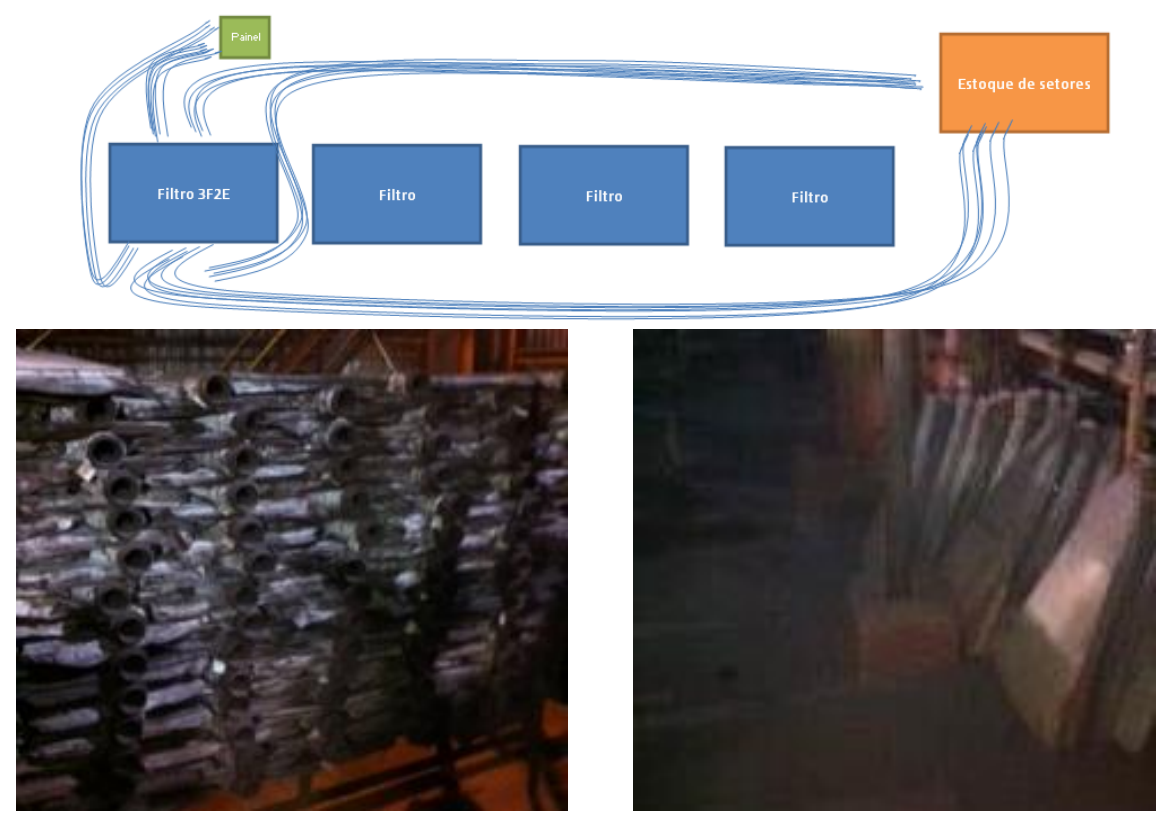

Figura 6. Disposição dos setores dos filtros.

Situação Proposta: Reduzir a quantidade de movimentações e aumentar produtividade na troca de setores. Ganho em média de 2 min para buscar e 2 para levar setores / filtro. Criando estoques intermediários próximos aos filtros.
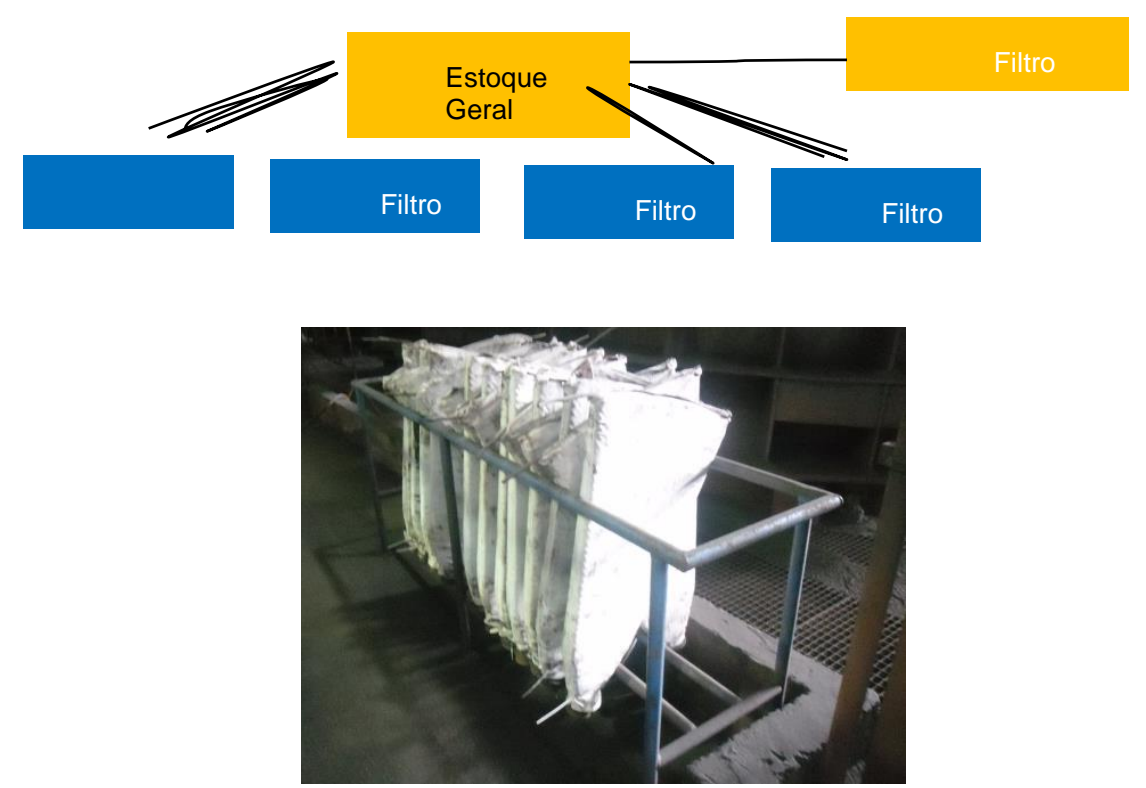

Figura 7. Disposição do estoque intermediário dos setores dos filtros.

Os dados abaixo demonstram elevação do rendimento operacional das filtragens, para as usinas Itabrasco e Hispanobrás.

\footnotetext{
* Contribuição técnica ao 44 Seminário de Redução de Minério de Ferro e Matérias-primas, Simpósio Brasileiro de Minério de Ferro e 2ํㅗ Simpósio Brasileiro de Aglomeração de Minério de Ferro, 15 a 18 de setembro de 2014, Belo Horizonte, MG, Brasil.
} 
Simpósio de Administração da produção, logística e operações internacionais (Simpoi), 9,2006, São Paulo. Anais... São Paulo: FGV-EAESP,

4 Stevenson WJ. Administração das operações de produção. 6.ed. Rio de Janeiro: LTC, 2001.

5 Ohno T. O sistema Toyota de produção: além da produção em larga escala. Porto Alegre: Bookman,1997.

6 Womack JP, Jones DT. A máquina que mudou o mundo. Rio de Janeiro: Elsevier, 2004.

7 Flynn BB, Schroeder RG, Flynn EJ, Sakakibara S, Bates KA. World-class manufacturing project: overview and selected results. International Journal of Operations \& Production Management, 1997;17(7): 671-685. 